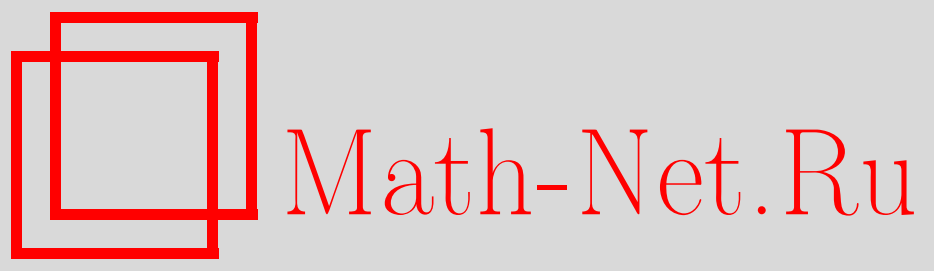

Е. А. Потехина, Приложение произведения Адамара к некоторым комбинаторным и вероятностным задачам, Дискрет. матем., 2016, том 28, выпуск 1, 101-112

DOI: https://doi.org/10.4213/dm1360

Использование Общероссийского математического портала Math-Net.Ru подразумевает, что вы прочитали и согласны с пользовательским соглашением http://www . mathnet.ru/rus/agreement

Параметры загрузки:

IP : 54.198 .64 .247

26 апреля 2023 г., 14:53:19

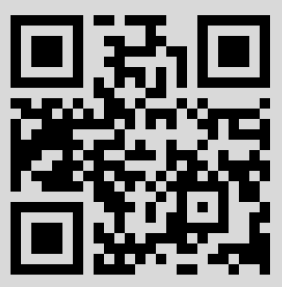




\title{
Приложение произведения Адамара к некоторым комбинаторным и вероятностным задачам
}

\author{
() 2016 г. Е. А. Потехина*
}

\begin{abstract}
Представлено усовершенствование алгебраического метода вычисления произведения Адамара рациональных функций и некоторые приложения произведения Адамара к комбинаторным и вероятностным задачам.
\end{abstract}

Ключевые слова: произведение Адамара, производящие функции, рациональные степенные ряды

\section{Введение}

В статье рассматриваются новые приемы вычисления произведения Адамара рациональных функций, позволяющие находить решения некоторых комбинаторных и вероятностных задач в явном виде.

Произведением Адамара формальных степенных рядов $G(x)=\sum_{k=0}^{\infty} g_{k} x^{k}$ и $H(x)=\sum_{k=0}^{\infty} h_{k} x^{k}$ называется степенной ряд $G(x) * H(x)=\sum_{k=0}^{\infty} g_{k} h_{k} x^{k}$.

Помимо задач перечислительной комбинаторики и дискретной теории вероятностей в научной литературе можно встретить применения произведения Адамара в задачах комплексного анализа ([1] - [3]), линейного программирования [4], математической физики ([5], [6]).

Хорошо известно, что произведение Адамара разложений в ряд рациональных функций является рациональной функцией ([7, с. 308], [8, с. 55]). Вопрос о рациональности произведения Адамара рациональных функций нескольких переменных исследовал Е.К. Лейнартас [3]. Исследованием многомерного произведения Адамара занимались также Л.А. Айзенберг [1], М.М. Елин [2], В.П. Кривоколеско [9]. В то же время, явных формул для вычисления произведения Адамара степенных рядов рациональных функций в общем случае до недавнего времени известно не было.

Вычисление произведения Адамара может быть сведено к вычислению $\Omega$-оператора П.А. Мак-Магона, введенного им в комбинаторной теории разбиений целых чисел (см. [10, с. 84]). Работа [11] посвящена построению алгоритма вычисления $\Omega$-оператора Мак-Магона для случая рациональных функций. Этот алгоритм

* Место работы: Череповецкий государственный университет, е-mail: peajk7@mail.ru 
может быть применен для вычисления произведения Адамара. Алгоритм использует разложение рациональных функций на простейшие дроби и технику симметрических функций для отыскания $\Omega$-оператора от произведений простейших дробей. Вычисление $\Omega$-оператора представляет интерес в связи с рядом комбинаторных задач, которые могут быть решены с его применением. Много таких задач рассмотрено в серии работ Г. Эндрюса с соавторами, подробный список которых приведен в [11].

Вероятностную интерпретацию серии тождеств, полученных с использованием произведения Адамара, дают в [9] Е.К. Лейнартас и В.П. Кривоколеско. В [12] рассмотрены приложения произведения Адамара к вычислению производящих функций распределений некоторых статистик от серий в последовательностях 1-зависимых индикаторов.

Конкретные комбинаторные и вероятностные задачи, с одной стороны, служат источником примеров, в которых требуется вычислять произведение Адамара степенных рядов, а с другой - их решение позволяет находить новые приёмы вычисления произведений Адамара рациональных функций в более или менее общих ситуациях.

В статье предложены новые приемы вычисления произведений Адамара рациональных функций. Известный ранее алгебраический метод [13] вычисления произведений Адамара рациональных функций требует вычисления определителей порядка $m+n$. С помощью методов комбинаторного анализа и линейной алгебры порядок определителей автором снижен до величины $\min (m, n)$, что расширяет возможности применения произведения Адамара для получения решения конкретных задач в явном виде. Представлено приложение произведения Адамара к вычислению производящих функций распределений некоторых статистик от серий рекуррентных событий. Ранее произведение Адамара в исследованиях распределений таких статистик не применялось. Применение произведения Адамара позволяет явно вычислить производящие функции распределений некоторых характеристик случайных последовательностей в некоторых случаях, когда их явное вычисление с помощью комбинаторных методов затруднительно.

\section{1. Перечисление замощений прямоугольника плит- ками}

Рассмотрим производящую функцию

$$
\sum_{r=0}^{\infty} f_{r}\left(d_{1}, d_{2}, \ldots, d_{n}\right) x^{r}=\left(1-d_{1} x-d_{2} x^{2}-\ldots-d_{n} x^{n}\right)^{-1}
$$

последовательности, задаваемой рекуррентным соотношением

$$
f_{r}\left(d_{1}, d_{2}, \ldots, d_{n}\right)=d_{1} f_{r-1}+d_{2} f_{r-2}+\ldots+d_{n} f_{r-n}
$$

с начальными условиями $f_{0}=1$ и $f_{r}=0$ при $r<0$. При $d_{i}=1(i=1,2, \ldots, n)$ и $n=2$ данная последовательность представляет собой последовательность чисел Фибоначчи. Элемент $f_{r}\left(d_{1}, d_{2}, \ldots, d_{n}\right)$ можно интерпретировать как сумму весов всех возможных замощений прямоугольника размера $1 \times r$ плитками размеров $1 \times 1,1 \times 2$, 
$\ldots, 1 \times n$ с весами $d_{1}, d_{2}, \ldots, d_{n}$ соответственно. Под весом замощения понимается произведение весов образующих его плиток.

При $r=4$ прямоугольник размера $1 \times 4$ можно замостить плитками размеров $1 \times 1,1 \times 2$ и $1 \times 3$ семью способами. Сумма весов этих замощений определяется равенством $f_{4}\left(d_{1}, d_{2}, d_{3}\right)=d_{1}^{4}+3 d_{1}^{2} d_{2}+2 d_{1} d_{3}+d_{2}^{2}$.

Коэффициент $f_{r}\left(d_{1}, d_{2}, \ldots, d_{n}\right) f_{r}\left(b_{1}, b_{2}, \ldots, b_{m}\right)$ при $x^{r}$ в произведении

$$
\begin{gathered}
\left(1-d_{1} x-d_{2} x^{2}-\ldots-d_{n} x^{n}\right)^{-1} *\left(1-b_{1} x-b_{2} x^{2}-\ldots-b_{m} x^{m}\right)^{-1}= \\
=\sum_{r=0}^{\infty} f_{r}\left(d_{1}, d_{2}, \ldots, d_{n}\right) f_{r}\left(b_{1}, b_{2}, \ldots, b_{m}\right) x^{r}
\end{gathered}
$$

можно интерпретировать как сумму весов всех замощений прямоугольника размера $2 \times r$ плитками размеров $1 \times 1,1 \times 2, \ldots, 1 \times n$ в верхнем ряду, а также плитками размеров $1 \times 1,1 \times 2, \ldots, 1 \times m-$ в нижнем ряду. Плитка верхнего ряда размера $1 \times i$ имеет вес $d_{i}(i=1,2, \ldots, n)$. Плитка нижнего ряда размера $1 \times j$ имеет вес $b_{j}$ $(j=1,2, \ldots, m)$.

До недавнего времени задача перечисления замощений плитками прямоугольника размера $2 \times r$ была решена лишь для случаев, сводящихся к вычислению произведения Адамара вида

$$
\left(1-a x-b x^{m}\right)^{-1} *\left(1-c x-d x^{n}\right)^{-1} .
$$

Это произведение Адамара вычислено комбинаторным методом в [14] при $n=2$, $b=1, d=1$. Метод указанной работы не применим при $n>2$.

Общее решение задачи перечисления замощений плитками прямоугольника размера $2 \times r$ можно получить с помощью алгебраического метода вычисления произведения Адамара, предложенного в [13]. Непосредственное применение алгебраического метода для решения указанной задачи требует вычисления определителей порядка $m+n$. В этой работе с помощью методов комбинаторного анализа и линейной алгебры порядок определителей автором снижен на величину $m$. А именно, верно следующее утверждение.

Теорема 1. Для любых иельх $m, n, k(m \geqslant 2, n \geqslant 2, k<m)$ справедлива формула

$$
\frac{1}{1-d_{1} x-d_{2} x^{2}-\ldots-d_{n} x^{n}} * \frac{x^{k}}{1-b_{1} x-b_{2} x^{2}-\ldots-b_{m} x^{m}}=\frac{\operatorname{det} \mathbf{G}}{\operatorname{det} \mathbf{H}},
$$

где $\mathbf{H}$ - матрица порядка п, имеющая вид

$$
\left(\begin{array}{cccccc}
D_{1} & L_{12} & L_{13} & \ldots & L_{1, n-1} & L_{1, n} \\
D_{2} & 1+L_{22} & L_{23} & \ldots & L_{2, n-1} & L_{2, n} \\
D_{3} & -b_{1}+L_{32} & 1+L_{33} & \ldots & L_{3, n-1} & L_{3, n} \\
\ldots & \ldots & \ldots & \ldots & \ldots & \ldots \\
D_{n-1} & -b_{n-3}+L_{n-1,2} & -b_{n-4}+L_{n-1,3} & \ldots & 1+L_{n-1, n-1} & L_{n-1, n} \\
D_{n} & -b_{n-2}+L_{n, 2} & -b_{n-3}+L_{n, 3} & \ldots & -b_{1}+L_{n, n-1} & 1+L_{n, n}
\end{array}\right)
$$

$L_{i j}=-\sum_{s=i}^{m} b_{s} x^{s+j-i} \sum_{t=j}^{s+j-i} d_{t} f_{s-t+j-i}, D_{i}=-\sum_{s=0}^{m-i+1} b_{s+i-1} f_{s} x^{s} \quad(i=1,2, \ldots, n$, $j=2,3, \ldots, n), b_{0}=-1$, 


$$
f_{s}=f_{s}\left(d_{1}, d_{2}, \ldots, d_{n}\right)= \begin{cases}d_{1} f_{s-1}+d_{2} f_{s-2}+\ldots+d_{n} f_{s-n} & \text { nрu } s>0 \\ 1 & \text { nрu } s=0 \\ 0 & \text { nри } s<0\end{cases}
$$

а матрица $\mathbf{G}$ получается из $\mathbf{H}$ заменой первой строки строкой

$$
\left(\begin{array}{llllll}
C_{1} & C_{2} & C_{3} & \cdots & C_{n-1} & C_{n}
\end{array}\right),
$$

где $C_{i}=x^{k+i-1} \sum_{s=i}^{n} d_{s} f_{k-s+i-1}$.

Доказательство. Как следует из теоремы 1 [13], для произведения Адамара справедливо представление

$$
\frac{1}{1-d_{1} x-d_{2} x^{2}-\ldots-d_{n} x^{n}} * \frac{x^{k}}{1-b_{1} x-b_{2} x^{2}-\ldots-b_{m} x^{m}}=\frac{P(x)}{Q(x)},
$$

в котором функция $Q(x)=\operatorname{det} \mathbf{Q}$, где $\mathbf{Q}=\left(q_{i j}\right)_{m+n}-$ такая матрица порядка $m+n$, что

$$
q_{i j}= \begin{cases}1 & \text { при } i=j, \\ -d_{j-i} x^{j-i} & \text { при } 1 \leqslant i \leqslant m, 1 \leqslant j-i \leqslant n, \\ -b_{i-j} & \text { при } m+1 \leqslant i \leqslant m+n, 1 \leqslant i-j \leqslant m, \\ 0 & \text { в остальных случаях; }\end{cases}
$$

а функция $P(x)=\operatorname{det} \mathbf{P}$, где $\mathbf{P}=\left(p_{i j}\right)_{m+n}$, причем $\mathbf{P}$ получается из $\mathbf{Q}$ заменой $m$-й строки строкой, в которой $(m-k)$-й элемент равен единице, а все остальные элементы равны нулю.

Умножим матрицу $\mathbf{Q}$ слева на матрицу $\widetilde{\mathbf{Q}}=\left(\widetilde{q}_{i j}\right)_{m+n}$, в которой

$$
\widetilde{q}_{i j}= \begin{cases}1 & \text { при } i=j, \\ \sum_{s=i-j}^{n} b_{s} f_{s-i+j} x^{s-i+j} & \text { при } m+1 \leqslant i \leqslant m+n, 1 \leqslant j \leqslant m, \\ 0 & \text { в остальных случаях. }\end{cases}
$$

При этом воспользуемся рекуррентным соотношением (1). Поскольку $\widetilde{\mathbf{Q}}-$ нижняя треугольная матрица, элементы главной диагонали которой равны 1 , то $\operatorname{det} \widetilde{\mathbf{Q}}=1$. Следовательно,

$$
\operatorname{det}(\widetilde{\mathbf{Q}} \mathbf{Q})=\operatorname{det} \widetilde{\mathbf{Q}} \cdot \operatorname{det} \mathbf{Q}=\operatorname{det} \mathbf{Q}=Q(x)
$$

Получаем

$$
Q(x)=\operatorname{det}(\widetilde{\mathbf{Q}} \mathbf{Q})=\left|\begin{array}{cc}
\mathbf{S}_{m} & \mathbf{T} \\
\mathbf{0} & \mathbf{H}
\end{array}\right|,
$$

где $\mathbf{T}$ - матрица размерности $m \times n, \mathbf{S}_{m}$ - верхняя треугольная матрица порядка $m$, элементы главной диагонали которой равны 1, $\mathbf{0}$ - нулевая матрица размерности $n \times m, \mathbf{H}-$ матрица, указанная в формулировке теоремы. Отсюда находим

$$
Q(x)=\operatorname{det} \mathbf{S}_{m} \cdot \operatorname{det} \mathbf{H}=1^{m} \cdot \operatorname{det} \mathbf{H}=\operatorname{det} \mathbf{H} .
$$

Аналогично, умножим матрицу $\mathbf{P}$ слева на матрицу $\widetilde{\mathbf{P}}=\left(\widetilde{p}_{i j}\right)_{m+n}$, в которой

$$
\widetilde{p}_{i j}= \begin{cases}1 & \text { при } i=j, \\ -f_{j-m+k} x^{j-m+k} & \text { при } i=m, m-k \leqslant j \leqslant m-1, \\ \sum_{s=i-j}^{n} b_{s} f_{s-i+j} x^{s-i+j} & \text { при } m+1 \leqslant i \leqslant m+n, 1 \leqslant j \leqslant m-1, \\ 0 & \text { в остальных случаях. }\end{cases}
$$


Легко видеть, что $\operatorname{det} \widetilde{\mathbf{P}}=1$. Следовательно, $P(x)=\operatorname{det}(\widetilde{\mathbf{P}} \mathbf{P})$. Разложив $\operatorname{det}(\widetilde{\mathbf{P}} \mathbf{P})$ по последнему столбцу, получаем определитель, равный $\operatorname{det}(\widetilde{\mathbf{P}} \mathbf{P})$ и имеющий порядок $m+n-1$. Таким образом, имеем

$$
P(x)=\operatorname{det}(\widetilde{\mathbf{P}} \mathbf{P})=\left|\begin{array}{cc}
\mathbf{V}_{m-1} & \mathbf{W} \\
\mathbf{0} & \mathbf{G}
\end{array}\right|,
$$

где $\mathbf{V}_{m-1}$ - верхняя треугольная матрица порядка $m-1$, элементы главной диагонали которой равны $1, \mathbf{W}$ - матрица размерности $(m-1) \times n, \mathbf{0}$ - нулевая матрица размерности $n \times(m-1), \mathbf{G}$ - матрица, указанная в формулировке теоремы. Отсюда находим

$$
P(x)=\operatorname{det} \mathbf{V}_{m-1} \cdot \operatorname{det} \mathbf{G}=1^{m-1} \cdot \operatorname{det} \mathbf{G}=\operatorname{det} \mathbf{G} .
$$

Если $n>m$, то для решения указанной выше задачи целесообразно воспользоваться следующей теоремой.

Теорема 2. Для любых иелых $m, n, k(m \geqslant 2, n \geqslant 2, k<m)$ справедлива формула

$$
\frac{1}{1-d_{1} x-d_{2} x^{2}-\ldots-d_{n} x^{n}} * \frac{x^{k}}{1-b_{1} x-b_{2} x^{2}-\ldots-b_{m} x^{m}}=\frac{(-1)^{k} \operatorname{det} \mathbf{R}_{m, m-k}}{\operatorname{det} \mathbf{R}},
$$

где $\mathbf{R}$ - матрица порядка $m$ вида

$$
\left(\begin{array}{cccccc}
1+A_{11} & -d_{1} x+A_{12} & -d_{2} x^{2}+A_{13} & \ldots & -d_{m-2} x^{m-2}+A_{1, m-1} & B_{1} \\
A_{21} & 1+A_{22} & -d_{1} x+A_{23} & \ldots & -d_{m-3} x^{m-3}+A_{2, m-1} & B_{2} \\
A_{31} & A_{32} & 1+A_{33} & \ldots & -d_{m-4} x^{m-4}+A_{3, m-1} & B_{3} \\
\ldots & \ldots & \ldots & \ldots & \ldots & \ldots \\
A_{m-1,1} & A_{m-1,2} & A_{m-1,3} & \ldots & 1+A_{m-1, m-1} & B_{m-1} \\
A_{m, 1} & A_{m, 2} & A_{m, 3} & \ldots & A_{m, m-1} & B_{m}
\end{array}\right),
$$

$A_{i j}=-\sum_{s=m-i+1}^{n} d_{s} x^{s} \sum_{t=m-j+1}^{m} b_{t} f_{s-t+i-j}, B_{i}=-\sum_{s=m-i}^{n} f_{s-m+i} d_{s} x^{s} \quad(i=1$, $2, \ldots, m, j=1,2, \ldots, m-1), d_{0}=-1$,

$$
f_{s}=f_{s}\left(b_{1}, b_{2}, \ldots, b_{m}\right)= \begin{cases}b_{1} f_{s-1}+b_{2} f_{s-2}+\ldots+b_{m} f_{s-m} & \text { nрu } s>0 \\ 1 & \text { nрu } s=0 \\ 0 & \text { nрu } s<0\end{cases}
$$

матрица $\mathbf{R}_{m, m-k}$ получается из $\mathbf{R}$ вычеркиванием $m$-й строки $u(m-k)$-го столбиа.

Теорема 2 доказывается аналогично теореме 1.

Таким образом, теоремы 1 и 2 дают новый прием вычисления произведений Адамара рациональных функций вида

$$
\frac{1}{1-d_{1} x-d_{2} x^{2}-\ldots-d_{n} x^{n}} * \frac{x^{k}}{1-b_{1} x-b_{2} x^{2}-\ldots-b_{m} x^{m}},
$$

где $m, n, k \in \mathbb{Z}(m \geqslant 2, n \geqslant 2,0 \leqslant k \leqslant m)$, сводящийся к вычислению определителей, имеющих порядок $\min (m, n)$. Известный ранее алгебраический метод [13] вычисления произведений Адамара рациональных функций указанного вида требует 
вычисления определителей порядка $m+n$. Посредством теорем 1 и 2 задача перечисления замощений прямоугольника размера $2 \times r$ плитками произвольной длины решена в общем виде. Полученный в статье новый прием вычисления произведения Адамара рациональных функций позволяет в данной задаче снять ограничения на длину плитки и решить известную ранее задачу в новой постановке. Теоремы 1 и 2 дают явные формулы для вычисления произведения Адамара рациональных функций. Они применимы для правильных рациональных дробей (при $k<m$ ). В общем случае можно выделить целую часть дроби, применить свойство линейности произведения Адамара, а также формулу $x^{k} * \sum_{s=0}^{\infty} g_{s} x^{s}=g_{k} x^{k}$.

\section{2. Осциллирующая последовательность в задаче пе- речисления замощений прямоугольника плитками по числу типов взаимного расположения плиток}

Рассмотрим задачу замощения прямоугольника плитками в динамике. Будем укладывать верхний ряд бесконечной полосы ширины 2 плитками размеров $1 \times 1,1 \times 2, \ldots$, $1 \times n$ и весами $d_{1}, d_{2}, \ldots, d_{n}$ соответственно, а нижний ряд - плитками размеров $1 \times 1$, $1 \times 2, \ldots, 1 \times m$ и весами $b_{1}, b_{2}, \ldots, b_{m}$ соответственно.

Укладку верхнего ряда будем выполнять последовательно слева направо до тех пор, пока верхний ряд не окажется длиннее нижнего. Затем выполняем укладку нижнего ряда последовательно слева направо до тех пор, пока нижний ряд не окажется длиннее верхнего. Таким образом, чередуя укладку верхнего и нижнего рядов, заполняем весь прямоугольник. В случае, когда длины верхнего и нижнего рядов равны, будем полагать, что нижний ряд длиннее, и переходить к укладке верхнего ряда.

В зависимости от того, длиннее верхний ряд или нижний, любую плитку можно отнести к одному из четырех типов: НН, НВ, ВН, ВВ. Плитку верхнего ряда назовем плиткой типа НВ, если при ее укладке выступающим становится верхний ряд. Плитку нижнего ряда назовем плиткой типа ВВ, если при ее укладке более длинным остается верхний ряд. Если же при укладке плитки нижнего ряда длиннее становится нижний ряд, то эту плитку отнесем к плиткам типа ВН. Плитку верхнего ряда назовем плиткой типа НН, если при ее укладке более длинным остается нижний ряд. Под весом замощения понимается произведение весов образующих его плиток.

Вычислим производящую функцию

$$
F\left(x, u_{\mathrm{HH}}, u_{\mathrm{HB}}, u_{\mathrm{BH}}, u_{\mathrm{BB}}\right)=\sum_{\substack{k=0 \\ i_{1}+i_{2}+i_{3}+i_{4}=k}}^{\infty} w_{k, i_{1}, i_{2}, i_{3}, i_{4}} u_{\mathrm{HH}}^{i_{1}} u_{\mathrm{HB}}^{i_{2}} u_{\mathrm{BH}}^{i_{3}} u_{\mathrm{BB}}^{i_{4}} x^{k}
$$

где $w_{k, i_{1}, i_{2}, i_{3}, i_{4}}$ - сумма весов замощений прямоугольников всевозможных размеров $k$ плитками, из которых $i_{1}$ плиток типа НH, $i_{2}$ плиток типа $\mathrm{HB}, i_{3}$ плиток типа $\mathrm{BH}$, $i_{4}$ плиток типа ВВ. Для решения данной задачи воспользуемся методами теории графов и понятием осциллирующей последовательности. 
Определение 1. Пусть $\left(x_{k}\right)_{k=1}^{\infty},\left(y_{k}\right)_{k=1}^{\infty}-$ последовательности неотрицательных целых чисел. Последовательность целых чисел $\left(z_{k}\right)_{k=0}^{\infty}$ называют осииллирующей, если она удовлетворяет условиям

$$
z_{0}=0, \quad z_{k}=\left\{\begin{array}{ll}
z_{k-1}-y_{k} & \text { при } z_{k-1}>0, \\
z_{k-1}+x_{k} & \text { при } z_{k-1} \leqslant 0,
\end{array} \quad k>0 .\right.
$$

Будем полагать, что элементы последовательностей $\left(x_{k}\right)_{k=1}^{\infty}, \quad\left(y_{k}\right)_{k=1}^{\infty}$ принадлежат множествам $\{0,1, \ldots, n\},\{0,1, \ldots, m\}$ соответственно. Тогда элементы осциллирующей последовательности $\left(z_{k}\right)_{k=0}^{\infty}$ принадлежат множеству $\{-m+1,-m+2, \ldots, n-1, n\}$.

Рассмотрим ориентированный граф $G=(V, D)$ с множеством вершин

$$
V=\{-m+1,-m+2, \ldots, n-1, n\}
$$

и множеством дуг

$$
D=\left\{(i, j) \in V^{2} \mid i>0, j \leqslant i \text { или } i \leqslant 0, j \geqslant i\right\}
$$

Припишем дуге $(i, j)$ орграфа $G$ формальный вес

$$
w_{i j}= \begin{cases}d_{j-i} u_{\mathrm{HH}} & \text { при } i \leqslant 0, j \leqslant 0, \\ d_{j-i} u_{\mathrm{HB}} & \text { при } i \leqslant 0, j>0, \\ b_{i-j} u_{\mathrm{BH}} & \text { при } i>0, j \leqslant 0, \\ b_{i-j} u_{\mathrm{BB}} & \text { при } i>0, j>0 .\end{cases}
$$

Последовательность $\left(z_{k}\right)_{k=0}^{\infty}$ элементов множества $V$ является последовательностью вершин некоторого бесконечного пути в орграфе $G$ тогда и только тогда, когда она является осциллирующей.

Если в качестве последовательностей $\left(x_{k}\right)_{k=1}^{\infty},\left(y_{k}\right)_{k=1}^{\infty}$ рассматривать соответственно последовательности длин плиток верхнего и нижнего рядов замощения бесконечной полосы ширины 2 , то осциллирующая последовательность $\left(z_{k}\right)_{k=0}^{\infty}$ строится по описанному выше алгоритму укладки плиток. Здесь $z_{0}=0$ соответствует начальному моменту укладки, когда прямоугольник пуст.

Перенумеруем вершины определенного выше графа $G$ в возрастающем порядке первыми $m+n$ натуральными числами и рассмотрим матрицу формальных весов $\mathbf{A}=\left(a_{i j}\right)$ орграфа $G$. Таким образом,

$$
a_{i j}= \begin{cases}d_{j-i} u_{\mathrm{HH}} & \text { при } 1 \leqslant i \leqslant j \leqslant m, 0 \leqslant j-i \leqslant n, \\ d_{j-i} u_{\mathrm{HB}} & \text { при } 1 \leqslant i \leqslant m, m+1 \leqslant j \leqslant m+n, 1 \leqslant j-i \leqslant n, \\ b_{i-j} u_{\mathrm{BH}} & \text { при } m+1 \leqslant i \leqslant m+n, 1 \leqslant j \leqslant m, 1 \leqslant i-j \leqslant m, \\ b_{i-j} u_{\mathrm{BB}} & \text { при } m+1 \leqslant i \leqslant m+n, m+1 \leqslant j \leqslant m+n, 0 \leqslant i-j \leqslant m, \\ 0 & \text { в остальных случаях. }\end{cases}
$$

Пусть $b_{0}=0, d_{0}=0$. Для отыскания весов путей в орграфе $G$ известен метод трансфер-матрицы [7, с. 354-356], суть которого изложена в следующей лемме. 
Лемма 1. Пусть $h_{i j}(x)=\sum_{k=0}^{\infty} w_{i j}^{(k)} x^{k}-$ производящая функиия суммы весов путей длины $k$ в орграфе $G$, ведущих из вершины $i$ в вершину $j$. Тогда

$$
h_{i j}(x)=\frac{(-1)^{i+j} \operatorname{det}(\mathbf{E}-x \mathbf{A})_{j i}}{\operatorname{det}(\mathbf{E}-x \mathbf{A})},
$$

где $\mathbf{E}$ - единичная матриц, порядок которой равен числу вершин орграфа $G, \mathbf{A}-$ матрица весов орграфа $G, \operatorname{det}(\mathbf{E}-x \mathbf{A})_{j i}-$ определитель матриць, полученной из $(\mathbf{E}-x \mathbf{A})$ вычеркиванием $j$-й строки и $i$-го столбиа.

Непосредственное применение леммы 1 для решения задачи вычисления производящей функции весов замощений прямоугольника плитками требует вычисления определителя порядка $m+n$. Следующая теорема показывает, что порядок определителя, необходимого для вычисления производящей функции весов замощений прямоугольника плитками, можно уменьшить на $n$.

Теорема 3. Для любых иельх $m, n(m \geqslant 2, n \geqslant 2)$ справедлива формула

$$
F\left(x, u_{\mathrm{HH}}, u_{\mathrm{HB}}, u_{\mathrm{BH}}, u_{\mathrm{BB}}\right)=\frac{\operatorname{det} \mathbf{R}_{m, m}}{\operatorname{det} \mathbf{R}},
$$

где $\mathbf{R}$ - матрица порядка $\mathrm{m}$, имеющая вид

$$
\left(\begin{array}{cccccc}
1+A_{11} & D_{1}+A_{12} & D_{2}+A_{13} & \ldots & D_{m-2}+A_{1, m-1} & D_{m-1}+A_{1, m} \\
A_{21} & 1+A_{22} & D_{1}+A_{23} & \ldots & D_{m-3}+A_{2, m-1} & D_{m-2}+A_{2, m} \\
A_{31} & A_{32} & 1+A_{33} & \ldots & D_{m-4}+A_{3, m-1} & D_{m-3}+A_{3, m} \\
\ldots & \ldots & \ldots & \ldots & \ldots & \ldots \\
A_{m-1,1} & A_{m-1,2} & A_{m-1,3} & \ldots & 1+A_{m-1, m-1} & D_{1}+A_{m-1, m} \\
A_{m, 1} & A_{m, 2} & A_{m, 3} & \ldots & A_{m, m-1} & 1+A_{m, m}
\end{array}\right)
$$

$D_{i}=-d_{i} u_{\mathrm{HH}} x \quad(i=1,2, \ldots, m-1), A_{i j}=-u_{\mathrm{BH}} u_{\mathrm{HB}} x^{2} \sum_{s=m-i+1}^{n} d_{s} \times$ $\times \sum_{t=m-j+1}^{m} b_{t} f_{s-t+i-j}(i=1,2, \ldots, m, j=1,2, \ldots, m)$,

$f_{s}=f_{s}\left(b_{1}, b_{2}, \ldots, b_{m}, u_{\mathrm{BB}}, x\right)= \begin{cases}\left(b_{1} f_{s-1}+b_{2} f_{s-2}+\ldots+b_{m} f_{s-m}\right) u_{\mathrm{BB}} x & \text { nрu } s>0 \\ 1 & \text { nрu } s=0, \\ 0 & \text { nрu } s<0\end{cases}$

матрица $\mathbf{R}_{m, m}$ получается из $\mathbf{R}$ вычеркиванием $m$-й строки и

Доказательство. Как следует из вышесказанного, производящая функция

$$
F\left(x, u_{\mathrm{HH}}, u_{\mathrm{HB}}, u_{\mathrm{BH}}, u_{\mathrm{BB}}\right)=\left.h_{i j}(x)\right|_{i=0, j=0} .
$$

Если же вершины орграфа $G$ перенумеровать в возрастающем порядке первыми $m+n$ натуральными числами, то

$$
F\left(x, u_{\mathrm{HH}}, u_{\mathrm{HB}}, u_{\mathrm{BH}}, u_{\mathrm{BB}}\right)=\left.h_{i j}(x)\right|_{i=m, j=m} .
$$

В силу леммы 1 получаем

$$
F\left(x, u_{\mathrm{HH}}, u_{\mathrm{HB}}, u_{\mathrm{BH}}, u_{\mathrm{BB}}\right)=\frac{(-1)^{m+m} \operatorname{det}(\mathbf{E}-x \mathbf{A})_{m m}}{\operatorname{det}(\mathbf{E}-x \mathbf{A})}=\frac{\operatorname{det}(\mathbf{E}-x \mathbf{A})_{m m}}{\operatorname{det}(\mathbf{E}-x \mathbf{A})}=\frac{P(x)}{Q(x)} .
$$


Функция $Q(x)=\operatorname{det} \mathbf{Q}$, где $\mathbf{Q}=\left(q_{i j}\right)_{m+n}-$ матрица порядка $m+n$ с элементами

$$
q_{i j}= \begin{cases}1 & \text { при } i=j, \\ -d_{j-i} u_{\mathrm{HH}} x & \text { при } 1 \leqslant i<j \leqslant m, 1 \leqslant j-i \leqslant n, \\ -d_{j-i} u_{\mathrm{HB}} x & \text { при } 1 \leqslant i \leqslant m, m+1 \leqslant j \leqslant m+n, 1 \leqslant j-i \leqslant n, \\ -b_{i-j} u_{\mathrm{BH}} x & \text { при } m+1 \leqslant i \leqslant m+n, 1 \leqslant j \leqslant m, 1 \leqslant i-j \leqslant m, \\ -b_{i-j} u_{\mathrm{BB}} x & \text { при } m+1 \leqslant j<i \leqslant m+n, 1 \leqslant i-j \leqslant m, \\ 0 & \text { в остальных случаях. }\end{cases}
$$

Функция $P(x)=\operatorname{det} \mathbf{P}$, где $\mathbf{P}=\left(p_{i j}\right)_{m+n}$, причем $\mathbf{P}$ получается из $\mathbf{Q}$ заменой $m$-й строки строкой, в которой $m$-й элемент равен единице, а все остальные элементы равны нулю.

Умножим матрицу $\mathbf{Q}$ слева на матрицу $\widetilde{\mathbf{Q}}=\left(\widetilde{q}_{i j}\right)_{m+n}$ с элементами

$$
\widetilde{q}_{i j}= \begin{cases}1 & \text { при } i=j, \\ \sum_{s=j-i}^{n} f_{s-j+i} d_{s} u_{\mathrm{HB}} & \text { при } 1 \leqslant i \leqslant m, m+1 \leqslant j \leqslant m+n, \\ 0 & \text { в остальных случаях. }\end{cases}
$$

При этом воспользуемся рекуррентным соотношением (2). Поскольку $\widetilde{\mathbf{Q}}-$ верхняя треугольная матрица, элементы главной диагонали которой равны 1 , то $\operatorname{det} \widetilde{\mathbf{Q}}=1$. Следовательно,

$$
\operatorname{det}(\widetilde{\mathbf{Q}} \mathbf{Q})=\operatorname{det} \widetilde{\mathbf{Q}} \cdot \operatorname{det} \mathbf{Q}=\operatorname{det} \mathbf{Q}=Q(x)
$$

Получаем

$$
Q(x)=\operatorname{det}(\widetilde{\mathbf{Q}} \mathbf{Q})=\left|\begin{array}{cc}
\mathbf{R} & \mathbf{0} \\
\mathbf{T} & \mathbf{H}_{n}
\end{array}\right|
$$

где $\mathbf{T}$ - матрица размерности $n \times m, \mathbf{H}_{n}$ - нижняя треугольная матрица порядка $n$, элементы главной диагонали которой равны 1, $\mathbf{0}$ - нулевая матрица размерности $m \times n, \mathbf{R}-$ матрица, указанная в формулировке теоремы. Отсюда находим

$$
Q(x)=\operatorname{det} \mathbf{R} \cdot \operatorname{det} \mathbf{H}_{n}=\operatorname{det} \mathbf{R} \cdot 1^{n}=\operatorname{det} \mathbf{R} .
$$

Аналогично, умножим матрицу $\mathbf{P}$ слева на матрицу $\widetilde{\mathbf{P}}=\left(\widetilde{p}_{i j}\right)_{m+n}$ с элементами

$$
\widetilde{p}_{i j}= \begin{cases}1 & \text { при } i=j, \\ \sum_{s=j-i}^{n} f_{s-j+i} d_{s} u_{\mathrm{HB}} & \text { при } 1 \leqslant i \leqslant m-1, m+1 \leqslant j \leqslant m+n-1, \\ 0 & \text { в остальных случаях. }\end{cases}
$$

Легко видеть, что $\operatorname{det} \widetilde{\mathbf{P}}=1$. Следовательно, $P(x)=\operatorname{det}(\widetilde{\mathbf{P}} \mathbf{P})$. Получаем

$$
P(x)=\operatorname{det}(\widetilde{\mathbf{P}} \mathbf{P})=\left|\begin{array}{cc}
\mathbf{S}_{m} & \mathbf{0} \\
\mathbf{V} & \mathbf{L}_{n}
\end{array}\right|
$$

где $\mathbf{V}$ - матрица размерности $n \times m, \mathbf{L}_{n}-$ нижняя треугольная матрица порядка $n$, элементы главной диагонали которой равны $1, \mathbf{0}$ - нулевая матрица размерности $m \times n, \mathbf{S}_{m}$ - матрица порядка $m$, получающаяся из $\mathbf{R}$ заменой $m$-й строки строкой, в 
которой $m$-й элемент равен единице, а все остальные элементы равны нулю. Отсюда находим

$$
P(x)=\operatorname{det} \mathbf{S}_{m} \cdot \operatorname{det} \mathbf{L}_{n}=\operatorname{det} \mathbf{S}_{m} \cdot 1^{n}=\operatorname{det} \mathbf{S}_{m} .
$$

Применяя теорему Лапласа, раскладываем $\operatorname{det} \mathbf{S}_{m}$ по $m$-й строке. Получаем

$$
P(x)=\operatorname{det} \mathbf{S}_{m}=(-1)^{2 m} \operatorname{det} \mathbf{R}_{m, m}=\operatorname{det} \mathbf{R}_{m, m} .
$$

Таким образом, с помощью метода трансфер-матрицы здесь в общем виде решена задача вычисления производящей функции весов замощений прямоугольника плитками по числу типов взаимного расположения плиток. Объект исследования в данном случае является новым.

\section{3. Вероятность появления прямоугольника размера $k \times n$, состоящего из $m$ плиток, при замощении бес- конечной полосы ширины $k$}

Рассмотрим задачу замощения прямоугольника плитками в динамике. Будем укладывать бесконечную в одну сторону полосу ширины $k$ плитками размеров $1 \times 1$, $1 \times 2,1 \times 3, \ldots$, имеющими соответственно вероятности выпадения для первого ряда - $q_{11}, q_{12}, q_{13}, \ldots$, для второго ряда $-q_{21}, q_{22}, q_{23}, \ldots$ соответственно, и т.д., для $k$-го ряда $-q_{k 1}, q_{k 2}, q_{k 3}, \ldots$ соответственно.

Укладка плиток начинается с первого ряда пустой полосы и выполняется по следующему алгоритму. Каждая новая плитка укладывается в тот ряд, который в данный момент имеет наименьшую длину. Если несколько рядов плиток имеют одинаковую длину и она является наименьшей, то укладка выполняется в ряд с наименьшим номером. Таким образом, чередуя укладку рядов, заполняем полосу.

Пусть $\left(X_{1 j}\right)_{j=1}^{\infty},\left(X_{2 j}\right)_{j=1}^{\infty}, \ldots,\left(X_{k j}\right)_{j=1}^{\infty}$ - не зависящие друг от друга последовательности независимых одинаково распределённых случайных величин, принимающих неотрицательные целые значения,

$$
f_{i}(x)=\sum_{j=0}^{\infty} \mathbf{P}\left(X_{i j}=j\right) x^{j}=\sum_{j=0}^{\infty} q_{i j} x^{j} \quad(i=1,2, \ldots, k),
$$

здесь $q_{i j}$ - вероятность того, что плитка $i$-го ряда будет иметь длину $j$.

Рассмотрим производящую функцию

$$
F_{k}(x, t)=\sum_{n=0}^{\infty} \sum_{m=0}^{\infty} p_{k, n, m} x^{n} t^{m},
$$

где $p_{k, n, m}$ - вероятность того, что в случайном процессе на каком-то шаге появится прямоугольник размера $k \times n$, состоящий из $m$ плиток.

Справедлива следующая теорема.

Теорема 4. В принятых выше обозначениях справедливо равенство

$$
F_{k}(x, t)=\frac{1}{1-f_{1}(x) t} * \frac{1}{1-f_{2}(x) t} * \ldots * \frac{1}{1-f_{k}(x) t} .
$$


Доказательство. В силу независимости последовательностей $\left(X_{1 j}\right)_{j=1}^{\infty}$, $\left(X_{2 j}\right)_{j=1}^{\infty}, \ldots,\left(X_{k j}\right)_{j=1}^{\infty}$, а также ввиду того, что величины $X_{i j}$ - неотрицательные целочисленные, имеем

$$
\begin{aligned}
& F_{k}(x, t)=\sum_{n=0}^{\infty} \sum_{\substack{m=0 \\
i_{1}+i_{2}+\ldots+i_{k}=m}}^{\infty} \mathbf{P}\left(X_{11}+\ldots+X_{1 i_{1}}=n\right) \cdot \ldots \cdot \mathbf{P}\left(X_{k 1}+\ldots+X_{k i_{k}}=n\right) x^{n} t^{m}= \\
& =\sum_{n=0}^{\infty} \sum_{i_{1}=0}^{\infty} \ldots \sum_{i_{k}=0}^{\infty} \mathbf{P}\left(X_{11}+\ldots+X_{1 i_{1}}=n\right) \cdot \ldots \cdot \mathbf{P}\left(X_{k 1}+\ldots+X_{k i_{k}}=n\right) x^{n} t^{i_{1}+\ldots+i_{k}}= \\
& =\sum_{n=0}^{\infty}\left(\sum_{i_{1}=0}^{\infty} \mathbf{P}\left(X_{11}+\ldots+X_{1 i_{1}}=n\right) t^{i_{1}}\right) \ldots \cdot\left(\sum_{i_{k}=0}^{\infty} \mathbf{P}\left(X_{k 1}+\ldots+X_{k i_{k}}=n\right) t^{i_{k}}\right) x^{n}= \\
& =\left(\sum_{n=0}^{\infty}\left(\sum_{i_{1}=0}^{\infty} \mathbf{P}\left(X_{11}+\ldots+X_{1 i_{1}}=n\right) t^{i_{1}}\right) x^{n}\right) * \ldots \\
& \ldots *\left(\sum_{n=0}^{\infty}\left(\sum_{i_{k}=0}^{\infty} \mathbf{P}\left(X_{k 1}+\ldots+X_{k i_{k}}=n\right) t^{i_{k}}\right) x^{n}\right)= \\
& =\left(\sum_{i_{1}=0}^{\infty}\left(\sum_{n=0}^{\infty} \mathbf{P}\left(X_{11}+\ldots+X_{1 i_{1}}=n\right) x^{n}\right) t^{i_{1}}\right) * \ldots \\
& \ldots *\left(\sum_{i_{k}=0}^{\infty}\left(\sum_{n=0}^{\infty} \mathbf{P}\left(X_{k 1}+\ldots+X_{k i_{k}}=n\right) x^{n}\right) t^{i_{k}}\right) .
\end{aligned}
$$

Применяя свойство мультипликативности производящих функций, получаем

$$
\begin{gathered}
F_{k}(x, t)=\left(\sum_{i_{1}=0}^{\infty} f_{1}^{i_{1}}(x) t^{i_{1}}\right) *\left(\sum_{i_{2}=0}^{\infty} f_{2}^{i_{2}}(x) t^{i_{2}}\right) * \ldots *\left(\sum_{i_{k}=0}^{\infty} f_{k}^{i_{k}}(x) t^{i_{k}}\right)= \\
=\frac{1}{1-f_{1}(x) t} * \frac{1}{1-f_{2}(x) t} * \ldots * \frac{1}{1-f_{k}(x) t} .
\end{gathered}
$$

Таким образом, теорема 6 позволяет вычислить с применением произведения Адамара производящую функцию вероятностей того, что в случайном процессе на каком-то шаге появится прямоугольник размера $k \times n$, состоящий из $m$ плиток.

Информация о вероятности $p_{k, n, m}$ может быть использована при разработке алгоритмов распределения ресурсов в вычислительных сетях [16]. Под плитками будем понимать задачи, поступающие в вычислительную сеть, под длиной плитки - время, необходимое для решения данной задачи, под рядом плиток - последовательность задач, направляемых управляющим узлом сети конкретному вычислительному узлу. Тогда $p_{k, n, m}$ - вероятность того, что $k$ вычислительных узлов в момент $n$ завершат выполнение $m$ текущих задач. 


\section{Список литературы}

1. Айзенберг Л.А., Лейнартас Е.К., "Многомерная композиция Адамара и ядра Сеге", Сибирский математический журнал, 24:3 (1983), 3-10.

2. Елин М.М., "Многомерная композиция Адамара", Сибирский математический журнал, 35:35 (1994), 1052-1057.

3. Лейнартас Е.К., "Об одном обобщении произведения Адамара в $C^{n}$ ", Maтематические заметки, 32:4 (1982), 477-482; англ. пер.: Leinartas E. K., "Generalization of the Hadamard product in $C^{n}$ ", Math. Notes, 32:4 (1982), 718-721.

4. De Loera J.A., Haws D., Hemmecke R., Yoshida R., "A computational study of integer programming algorithms based on Barvinok's rational functions", Discrete Optimization, 2:2 (2005), 135-144.

5. Bostan A., Boukraa S., Christol G., Hassani S., Maillard J-M., "Using $n$-fold integrals as diagonals of rational functions and integrality of series expansions", J. Physics A: Math. and Theor., 46:18 85201 (2013).

6. Zhilinskii B., "Generating functions for effective Hamiltonians via the symmetrized Hadamard product", J. Physics A: Math. and Theor., 41:38 82004 (2008).

7. Stanley R., Enumerative Combinatorics, Wadsworth \& Brooks/Cole, 1984; пер. с англ.: Стенли Р., Перечислительная комбинаторика, Мир, Москва, 1990, 440 с.

8. Ландо С.К., Лекиии о производящих функииях, МЦНМО, Москва, 2007, 144 с.

9. Кривоколеско В.П., Лейнартас Е.К., "О тождествах с полиномиальными коэффициентами", Изв. Иркутского гос. ун-та. Сер. Матем., 5:3 (2012), 56-62.

10. Рыбников К.А., Комбинаторный анализ. Очерки истории, Изд-во мех.-матем. ф-та МГУ, Москва, 1996, 125 с.

11. Han G.-N., "A general algorithm for the MacMahon omega operator", Ann. Combinatorics, 7:4 (2003), 467-480.

12. Потехина Е.А., Толовиков М.И., "Распределение серий в последовательностях 1-зависимых индикаторов", Череповецкие научные чтения - 2010: Матер. Всерос. науч.-практ. конф. Ч. 3: Техн., естеств. и экон. науки, 2011, 136-139.

13. Потехина Е.А., Толовиков М.И., “Осциллирующее случайное блуждание и произведение Адамара рациональных функций”, Дискретнал математика, 25:3 (2013), 96-115; англ. пер.: Potekhina E. A., Tolovikov M. I., "Oscillating random walk and the Hadamard product of rational functions", Discrete Math. Appl., 24:1 (2014), 29-44.

14. Kim J.H., Hadamard products, lattice paths, and skew tableaux, $\mathrm{PhD}$ thesis, Brandeis Univ. Dep. Math. ProQuest, UMI Diss. Publ., 2013, 69 pp.

15. Feller W., An Introduction to Probability Theory and Its Applications, v. 1, 3rd edition, Wiley, 1968, 528 pp.; пер. с англ.: Феллер В., Введение в теорию вероятностей и ее приложения. В 2-х томах. Т. 1, Мир, Москва, 1984, 528 с.

16. Жук С.Н., "Об онлайн-алгоритмах упаковки прямоугольников в несколько полос", Дискретная математика, 19:4 (2007), 117-131; англ. пер.: Zhuk S. N., "On-line algorithms for packing rectangles into several strips", Discrete Math. Appl., 17:5 (2007), $517-531$. 